\title{
Rotational thromboelastometry for diagnosing sudden hyperfibrinolysis immediately after cardiopulmonary bypass during cardiac surgery
}

\author{
Joung-Min Kim ${ }^{1}$, Chungsik $\mathrm{Oh}^{2}$, Ju-Won Kim ${ }^{1}$, Hyun Ju Jung ${ }^{1}$, and Tae-Yop Kim² \\ ${ }^{1}$ Department of Anesthesiology and Pain Medicine, Uijongbu St. Mary's Hospital, The Catholic University of Korea, Uijongbu, \\ ${ }^{2}$ Department of Anesthesiology, Konkuk University Medical Center, Konkuk Universrity School of Medicine, Seoul, Korea
}

Coagulopathy is a frequent complication after prolonged use of cardiopulmonary bypass $(\mathrm{CPB})$ and usually requires transfusion of various blood components, including red blood cells, fresh frozen plasma (FFP), and platelets in cardiac surgery [1]. Considering the risk of transfusion-related complications and postoperative morbidity and mortality [2], accurate and fast determination of the whole-blood coagulation profile and the etiology of any associated coagulopathy is necessary to avoid unnecessary transfusions and/or to minimize their size [3]. Standard laboratory-based tests (SLTs) do not accurately reflect the whole-blood coagulation profile and unable to carry out timely and effective management of concurrent coagulopathies due to their relatively long turnaround times. Thus, implementation of a reliable and predictive intraoperative point-of-care (POC) coagulation test enables to diagnose various types of coagulopathy in a much faster and customized manner and facilitates intraoperative coagulation management by avoiding unnecessary transfusions, prompt transfusion of essential component(s) for managing concurrent coagulopathies, and prevent consumptive coagulopathy due to delayed transfusion in massive bleeding.

A 74-year-old male underwent an elective graft interposition procedure for a dilated ascending aorta (diameter $5.5 \mathrm{~cm}$ ). He had a history of aortic valve replacement surgery using a mechanical valve, and wrapping of the ascending aorta using a synthetic graft. He had been taking warfarin ( $5.5 \mathrm{mg}$ /daily), which had been switched to heparin 2 days before surgery. At the time at which warfarin was discontinued, his activated partial thromboplastin time (aPTT) and prothrombin time (PT) were prolonged, and his PT international normalization ratio (INR) was high; by the time of surgery, they had all decreased but were still beyond their normal ranges.

During 130 min-CPB with moderate hypothermia (rectal temperature, $28^{\circ} \mathrm{C}$ ), graft interposition of the dilated ascending aorta was underwent using synthetic graft. After achieving stable cardiovascular performance, as indicated by hemodynamic parameters including blood pressure, heart rate, central venous pressure, pulmonary arterial pressure, the thermodilution cardiac index, mixed vnous $\mathrm{O}_{2}$ saturation, and a routine transesophageal echocardiography examination. Immediately after weaning from CPB and protamine infusion, activated clotting time (ACT) and ROTEM (ROTEM, Tem International GmbH, Munich, Germany) assays including INTEM, EXTEM, FIBTEM, and APTEM were started as part of the routine coagulation management protocol at our institution. ACT was $144 \mathrm{~s}$ (the value before CPB was $167 \mathrm{~s}$ ), and until $20 \mathrm{~min}$ after starting the ROTEM assays, the tracings of ROTEM assays showed the following patterns: a slight reduction in the $\alpha$-angle in INTEM, EXTEM, and APTEM; prolonged clot formation time (CFT) in INTEM (67 s; reference value 30-110 s), EXTEM and APTEM (both $195 \mathrm{~s}$; reference values $34-159 \mathrm{~s}$ in both); and slight reductions in the amplitudes at $10 \mathrm{~min}$ (A10) and $20 \mathrm{~min}$ (A20) of INTEM (39 and $41 \mathrm{~mm}$; reference values $44-46 \mathrm{~mm}$ and $50-71 \mathrm{~mm}$, respec-

Corresponding author: Tae-Yop Kim, M.D., Ph.D., Department of Anesthesiology, Konkuk University Medical Center, Konkuk Universrity School of Medicine, 120-1, Neungdong-ro, Hwayang-dong, Gwangjin-gu, Seoul 143-729, Korea. Tel: 82-2-2030-5445, Fax: 82-2-2030-5449, E-mail: taeyop@gmail.com, pondkim@unitel.co.kr

(c) This is an open-access article distributed under the terms of the Creative Commons Attribution Non-Commercial License (http:// creativecommons.org/licenses/by-nc/3.0/), which permits unrestricted non-commercial use, distribution, and reproduction in any medium, provided the original work is properly cited. 
A

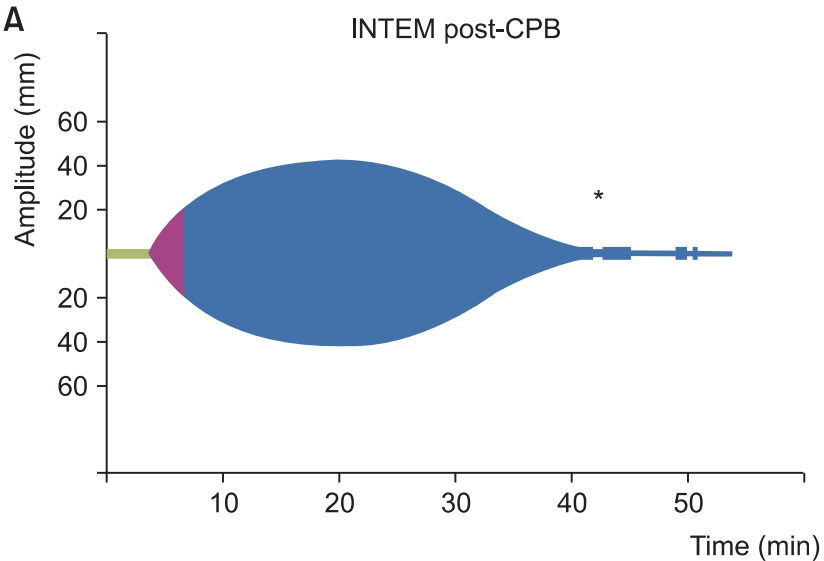

\section{C}

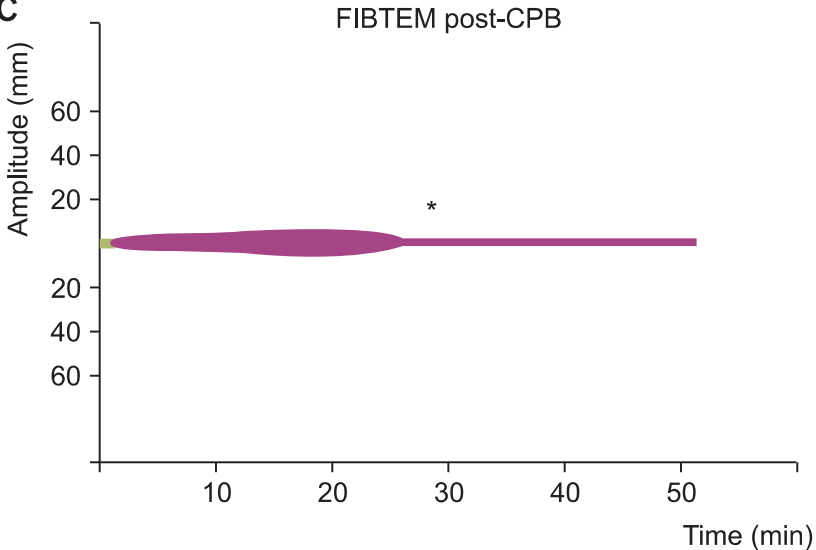

E

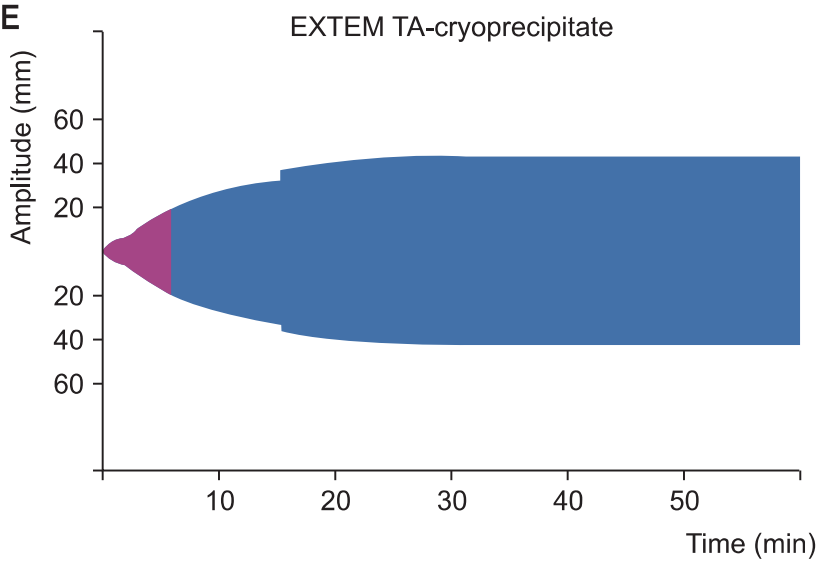

B

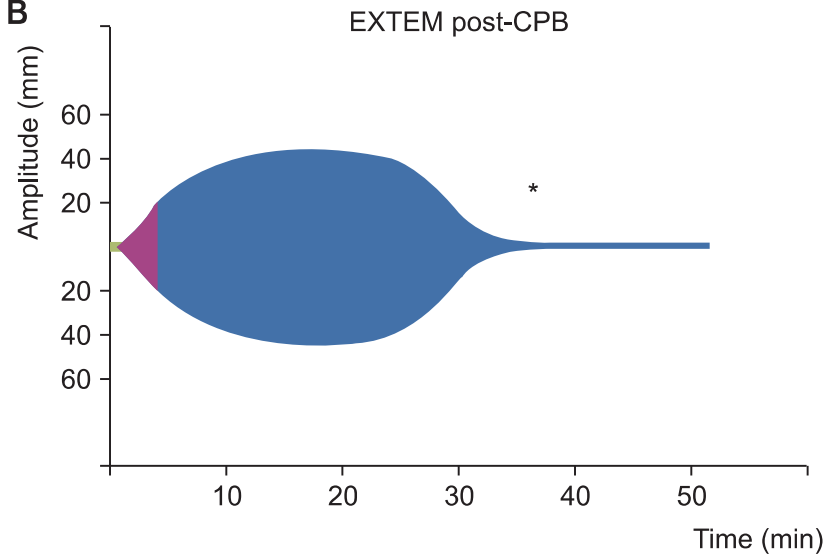

D

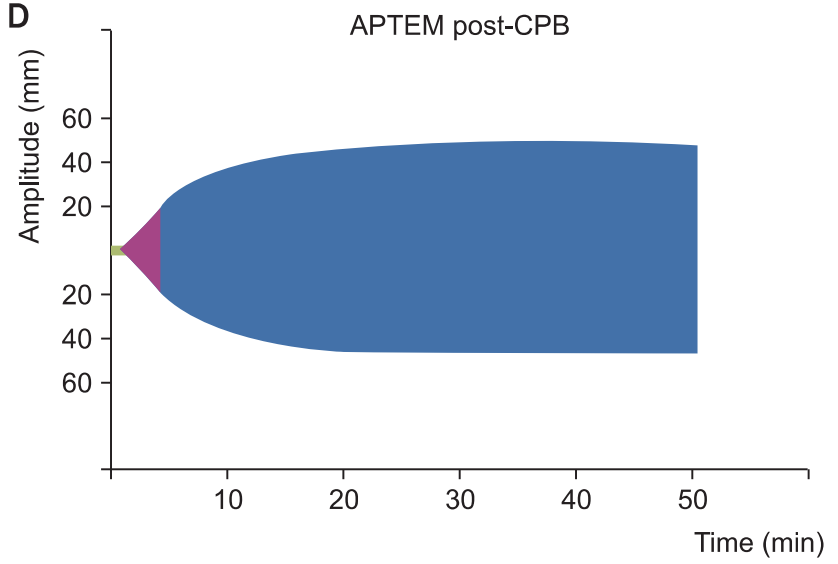

F

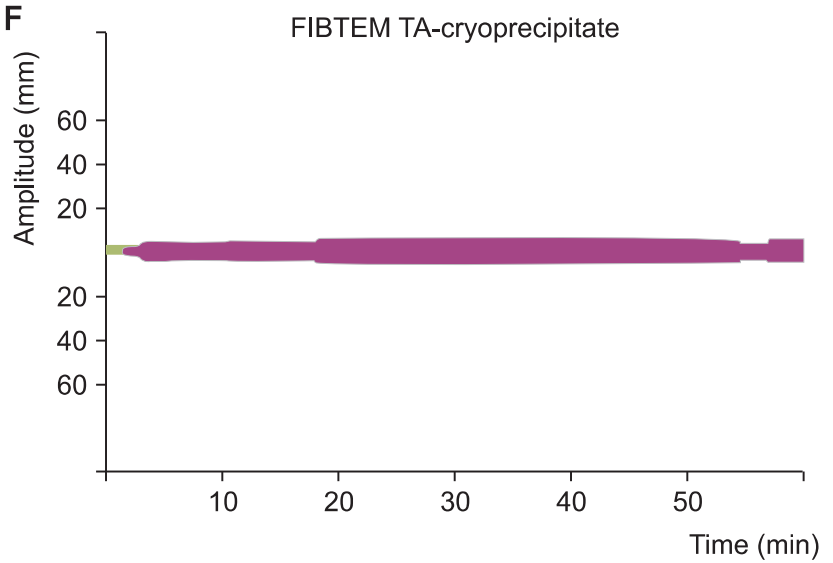

Fig. 1. Changes in multiple rotational thromboelastometry (ROTEM) assays. (A-D) INTEM, EXTEM, and APTEM post-CPB show a slight reduction in $\alpha$-angle $\left(61^{\circ}\right)$ and prolongations in CFT (67 s) in INTEM (reference values 70-83 and 30-110 s, respectively); a reduction in the $\alpha$-angles $\left(54^{\circ}\right.$ and $55^{\circ}$ ) and prolongation of CFT (195 and $200 \mathrm{~s}$ ) in EXTEM and APTEM (reference values 63-83 and 34-159 s, respectively); slight reductions in A10 (39, 39 , 39 mm) and A20 (41, 45, $49 \mathrm{~mm}$ ) in INTEM, EXTEM, and APTEM (reference values 44-46 mm and 50-71 mm, 43-65 mm and 50-72 $\mathrm{mm}$, and 43-65 mm and 50-72 $\mathrm{mm}$, respectively); and maximum clot formation (MCF, 4-6 mm) in FIBTEM (reference value $>7 \mathrm{~mm}$ ). Dramatic reductions of amplitudes (tails) suggesting hyperfibrinolysis with a slight deficiency of clotting factor, hypofibrinogemia, and thrombocytopenia were noted beyond 20 min after starting the ROTEM assays. An abrupt reduction of clot lysis indices at $30 \mathrm{~min}$ (CLI 30) in INTEM and EXTEM (38\% and 24\%; reference value 94-100\%) and their further worsening at 45 min (CLI 45) in INTEM and EXTEM (4\% and 2\%; reference value 94-100\%) were also noted. (E) After tranexamic acid (TA) administration, additional EXTEM assay showed restoration of normal clot lysis function, as indicated by normal CLI 30 and CLI 45, with continued clotting factor deficiency, hypofibrinogemia, and thrombocytopenia, as indicated by the reduced $\alpha$-angle and prolonged CFT and reduced amplitude (A10 and A20). (F) After completion of transfusions with fresh frozen plasma (2 units), platelet concentrate (300 ml), cryoprecipitate (8 units) and cryoprecipitate (6 units), additional FIBTEM assay around $50 \mathrm{~min}$ after TA administration suggested hypofibrinogenemia (MCF $<7 \mathrm{~mm})$. Post-CPB: immediately after weaning from cardiopulmonary bypass and protamine administration; TA-cryoprecipitate: after administering tranexamic acid ( 2 g) and cryoprecipitate (6 units); $\alpha$-angle: tangent of the slope between 2 and $20 \mathrm{~mm}$, CFT: clot formation time; A10 and A20: amplitudes $10 \mathrm{~min}$ and 20 min after the start of the assay; MCF: maximum clot firmness; CLI 30 and CLI 45: clot lysis indices (\% of clot strength) remaining 30 min and 45 min after clotting time (CT). 
tively), EXTEM and APTEM (39-45 and 39-49 mm; reference values 43-65 and 50-72 mm, respectively), and FIBTEM (4-6 $\mathrm{mm}$; reference value $>7 \mathrm{~mm}$ ). However, dramatic reductions in amplitudes, suggesting hyperfibrinolysis with a slight deficiency of clotting factor, hypofibrinogemia, and thrombocytopenia, were noted beyond 20 min after starting the ROTEM assays. Abrupt reductions in clot lysis indices at $30 \mathrm{~min}$ (CLI 30) were noted in INTEM and EXTEM (38\% and 24\%; reference value 94-100\%) as was their further worsening at $45 \mathrm{~min}$ (CLI 45) in INTEM and EXTEM (4\% and 2\%; reference value 94-100\%), producing "tails" (indicated by * in Figs. 1A-1C). The simultaneous APTEM tracing with a constant amplitude and no significant decrease in CLI 30 or CLI 45 (98-100\%; reference value 94-100\%) confirmed the hyperfibrinolytic status (Fig. 1D). At the same time, the amplitudes of ongoing INTEM, EXTEM, and FIBTEM tracings became undetectable due to complete resolution of clots by concurrent hyperfibrinolysis, and the assays were interrupted after about $50 \mathrm{~min}$ after they were started.

Based on these ROTEM assay results, $2.0 \mathrm{~g}$ tranexamic acid (TA) was administered intravenously and cryoprecipitate was added to transfusions of platelet concentrate and FFP. During the administration of TA and the thawing and preparation of the blood products, additional bleeding in the patient's nostrils was noted. In addition, the amount of bleeding, gradually increased. Hence, an additional EXTEM assay was initiated $10 \mathrm{~min}$ after the completion of TA administration; it showed restoration of normal clot lysis function, as indicated by normal CLI 30 and CLI 45, although clotting factor deficiency, hypofibrinogemia, and thrombocytopenia continued, as indicated by the reduced $\alpha$-angle and prolonged CFT and reduced amplitude (A10 and
A20) in EXTEM (Fig. 1E), likely due to ongoing massive bleeding.

After additional transfusions with FFP (2 units), platelet concentrate ( 8 units or about $300 \mathrm{ml}$ ), and cryoprecipitate ( 8 units), surgical bleeding was reduced significantly and the speed of intravascular volume resuscitation for maintaining stable hemodynamics became $10-15 \mathrm{ml} / \mathrm{min}$. An additional FIBTEM assay was initiated before transporting the patient to the intensive care unit (ICU) near the end of the surgery, around $50 \mathrm{~min}$ after TA administration. Cryoprecipitate (6 units) was transfused in the ICU based on the results of the final FIBTEM assay, suggesting hypofibrinogenemia (MCF $<7 \mathrm{~mm}$; Fig. $1 \mathrm{~F})$.

Severely bleeding patients need goal-directed coagulation management using a quick and reliable coagulation monitor as well as a targeted therapeutic approach specific to the test results [4]. Commonly used SLTs including PT, APTT, and fibrinogen assays are time-consuming. Furthermore, PT and aPTT tests do not reflect the underlying etiology of a complex coagulopathy [5]

Massive bleeding due to hyperfibrinolysis usually leads to further deterioration of coagulation function, because it is typically accompanied by further dilution of the plasma components responsible for maintaining coagulation, such as fibrinogen, clotting factors, and platelets. If the fibrinolytic condition was not diagnosed promptly in the present case, much greater consumptive bleeding, requiring a much greater amount of transfusion, would have persisted. The present case showed that the routine use of multiple ROTEM assays immediately after CPB enabled earlier and precise diagnosis of hyperfibrinolysis, which was superimposed on the usual CPB-induced bleeding diathesis, and timely anti-fibrinolytic therapy, using TA.

\section{References}

1. Bennett-Guerrero E, Zhao Y, O'Brien SM, Ferguson TB Jr, Peterson ED, Gammie JS, et al. Variation in use of blood transfusion in coronary artery bypass graft surgery. JAMA 2010; 304: 1568-75.

2. Hendrickson JE, Hillyer CD. Noninfectious serious hazards of transfusion. Anesth Analg 2009; 108: 759-69.

3. Koch CG, Li L, Duncan AI, Mihaljevic T, Loop FD, Starr NJ, et al. Transfusion in coronary artery bypass grafting is associated with reduced long-term survival. Ann Thorac Surg 2006; 81: 1650-7.

4. Geeraedts LM Jr, Demiral H, Schaap NP, Kamphuisen PW, Pompe JC, Frölke JP. 'Blind' transfusion of blood products in exsanguinating trauma patients. Resuscitation 2007; 73: 382-8.

5. Dzik WH. Predicting hemorrhage using preoperative coagulation screening assays. Curr Hematol Rep 2004; 3: 324-30. 